\title{
ARTICLE
}

\section{One-dimensional spherical ray-tracing extended to account for flat and cylindrical shields}

\author{
Jeffrey A. Favorite* \\ Los Alamos National Laboratory, Los Alamos, NM, 87545, USA
}

\begin{abstract}
It is often desirable to compute the uncollided component (direct from the source) of a detected particle flux using some form of ray-tracing, which in one-dimensional spherical geometries is extremely economical. A simple method for treating a flat shield exactly, within the context of spherical ray-tracing, is presented. A method for treating a cylindrical shield approximately, using the average value of the azimuthal angle for each value of the polar angle that is used in the spherical ray-tracing, is presented. The source remains spherical. The methods are verified numerically with a test problem that also shows the importance of these corrections.
\end{abstract}

\section{Keywords: uncollided gamma rays; ray-tracing; geometry transformation; sphere; box; cylinder}

\section{Introduction}

It is often desirable to compute the uncollided component (direct from the source) of a detected particle flux [1]. In many cases, the uncollided component might be the only part that is needed - for example, the scattering, background, and detector effects can be removed from a gamma-ray spectrum, leaving only the uncollided peaks. Ray-tracing is an important tool for determining the contribution of uncollided particles. We consider a one-dimensional spherical source-shield system of nested homogeneous materials with an external detector measuring the uncollided flux. Ray-tracing the uncollided flux through this geometry is extremely fast, requiring only a numerical integral over the polar angle between 0 (a ray from the detector to the object center) and the angle that subtends the outermost source radius [2]. The geometry is symmetric with respect to the azimuthal angle.

Spherical ray-tracing in this situation can easily be extended to account exactly for the case when the outermost shield layers are flat, rather than spherical. Spherical ray-tracing can be extended to account for the case when the outermost layers are cylindrical, rather than spherical, but only approximately because the real geometry is not symmetric with respect to the azimuthal angle.

This paper describes these extensions to spherical ray-tracing and presents numerical test problems.

\section{Sphere-to-plane correction}

Consider the system shown in Figure 1. The sphere will be converted to a plane (the "box") in the

*Corresponding author. Email: fave@lanl.gov ray-tracing code. The distance from the center of the sphere to the detector is $r_{d}$. The radius of the sphere is $r_{p}$. Draw a ray through the geometry from the detector point; the ray makes an angle $\theta$ with the line connecting the center of the sphere and the detector. Half of the track-length that the ray makes through the sphere is called $a$ and the extra track length that will be added when the sphere becomes a plane is called $b$. The distance from the detector point to the plane along the ray is called $c$. With these definitions, it is clear that

$$
\cos \theta=(b+a+c) / r_{d}
$$

and

$$
\cos \theta=\left(r_{d}-r_{p}\right) / c
$$

Rearranging Eq. (1) and using Eq. (2), rearranged for $c$, yields

$$
b+a=r_{d} \cos \theta-\frac{r_{d}-r_{p}}{\cos \theta}=\frac{r_{p}-r_{d} \sin ^{2} \theta}{\cos \theta}
$$

If $b$ is needed separately, it can be obtained by

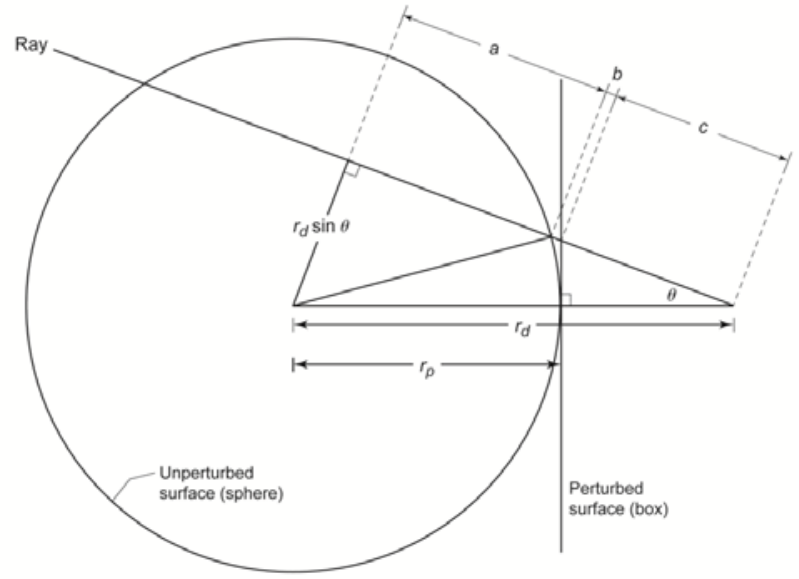

Figure 1. Spherical geometry in which the outer surface is to be treated as a plane in the ray-tracing. 
subtracting $a$, which, by the Pythagorean theorem, is

$$
a=\sqrt{r_{p}^{2}-r_{d}^{2} \sin ^{2} \theta}
$$

Thus, all that is needed is a flag to the spherical ray-tracing code that an input radius is actually meant to be treated as a plane. In general, this correction should only be applied in this way to the outer surfaces. An exterior multilayered flat shield may be treated in this way, but no flat surface should be inside a real spherical surface. Furthermore, because this method essentially makes the plane infinite, spherical sources must not be converted to flat sources in this manner.

\section{Sphere-to-cylinder correction}

Consider a Cartesian coordinate system with its origin on the axis of a right circular cylinder of radius $R$ and let that axis be coincident with the $z$ axis of the coordinate system. See Figure 2. Let $\overrightarrow{\mathbf{r}}_{\mathbf{d}}=x_{d} \hat{\mathbf{i}}+y_{d} \hat{\mathbf{j}}+z_{d} \hat{\mathbf{k}}$ be a vector from the origin to a point outside the cylinder (this point will be the detector point). Let unit vector $\hat{\mathbf{v}}=u \hat{\mathbf{i}}+v \hat{\mathbf{j}}+w \hat{\mathbf{k}}$ point in the direction of an arbitrary ray from $\overrightarrow{\mathbf{r}}_{\mathbf{d}}$ that intersects the cylinder, and let $g$ be the distance from $\overrightarrow{\mathbf{r}}_{\mathbf{d}}$ to the cylinder. Let $\overrightarrow{\mathbf{r}}_{\mathbf{c}}=x_{c} \hat{\mathbf{i}}+y_{c} \hat{\mathbf{j}}+z_{c} \hat{\mathbf{k}}$ be the vector from the origin to the point at which the ray crosses the cylinder. From Figure 2,

$$
\overrightarrow{\mathbf{r}}_{\mathbf{d}}+g \hat{\mathbf{v}}=\overrightarrow{\mathbf{r}}_{\mathbf{c}}
$$

or, taking the dot-product of both sides with $\hat{\mathbf{v}}$ and rearranging,

$$
g=\overrightarrow{\mathbf{r}}_{\mathbf{c}} \cdot \hat{\mathbf{v}}-\overrightarrow{\mathbf{r}}_{\mathbf{d}} \cdot \hat{\mathbf{v}}=\left|\overrightarrow{\mathbf{r}}_{\mathbf{c}}\right| \cos \alpha-\overrightarrow{\mathbf{r}}_{\mathbf{d}} \cdot \hat{\mathbf{v}}
$$

where $\alpha$ is the angle between the ray and $\overrightarrow{\mathbf{r}}_{\mathbf{c}}$ (Figure 2).

Applying the law of cosines for $\alpha$ yields

$$
\left|\overrightarrow{\mathbf{r}}_{\mathbf{d}}\right|^{2}=\left|\overrightarrow{\mathbf{r}}_{\mathbf{c}}\right|^{2}+g^{2}-2\left|\overrightarrow{\mathbf{r}}_{\mathbf{c}}\right| g \cos \alpha
$$

or, rearranging,

$$
\left|\overrightarrow{\mathbf{r}}_{\mathbf{c}}\right| \cos \alpha=\left(g^{2}+\left|\overrightarrow{\mathbf{r}}_{\mathbf{c}}\right|^{2}-\left|\overrightarrow{\mathbf{r}}_{\mathbf{d}}\right|^{2}\right) /(2 g)
$$

Using Eq. (8) in Eq. (6) and rearranging yields

$$
g^{2}+2\left(\overrightarrow{\mathbf{r}}_{\mathbf{d}} \cdot \hat{\mathbf{v}}\right) g+\left|\overrightarrow{\mathbf{r}}_{\mathbf{d}}\right|^{2}-\left|\overrightarrow{\mathbf{r}}_{\mathbf{c}}\right|^{2}=0
$$

Applying the Pythagorean theorem yields

$$
\left|\overrightarrow{\mathbf{r}}_{\mathrm{c}}\right|^{2}=z_{c}^{2}+R^{2}
$$

The direction cosine of the ray with respect to the $z$ axis is

$$
w=\left(z_{c}-z_{d}\right) / g
$$

rearrange this for $z_{c}$, use the result in Eq. (10), and use that result in Eq. (9) to find

$$
\begin{aligned}
g^{2}+2\left(\overrightarrow{\mathbf{r}}_{\mathbf{d}} \cdot \hat{\mathbf{v}}\right) g+\left|\overrightarrow{\mathbf{r}}_{\mathbf{d}}\right|^{2}- & \left(w^{2} g^{2}+2 w z_{d} g+z_{d}^{2}+R^{2}\right) \\
=0 &
\end{aligned}
$$

or, rearranging,

$$
\left(1-w^{2}\right) g^{2}+2\left(\overrightarrow{\mathbf{r}}_{\mathbf{d}} \cdot \hat{\mathbf{v}}-w z_{d}\right) g+\left|\overrightarrow{\mathbf{r}}_{\mathbf{d}}\right|^{2}-z_{d}^{2}-R^{2}=0
$$

Expand $\overrightarrow{\mathbf{r}}_{\mathbf{d}}$ and $\hat{\mathbf{v}}$ and simplify to find

$$
\left(1-w^{2}\right) g^{2}+2\left(u x_{d}+v y_{d}\right) g+x_{d}^{2}+y_{d}^{2}-R^{2}=0
$$

Now solve for $g$ :

$$
\begin{gathered}
g=\frac{-2\left(u x_{d}+v y_{d}\right)}{2\left(1-w^{2}\right)} \\
\pm \frac{\sqrt{4\left(u x_{d}+v y_{d}\right)^{2}-4\left(1-w^{2}\right)\left(x_{d}^{2}+y_{d}^{2}-R^{2}\right)}}{2\left(1-w^{2}\right)}
\end{gathered}
$$

Whether to use the plus or minus sign in Eq. (15) is determined next. The second term on the right side represents the (positive) distance from $\overrightarrow{\mathbf{r}}_{\mathbf{c}}$ to the point at which a radial line from the cylinder axis would be normal to the direction vector $\hat{\mathbf{v}}$; i.e., the length of line segment $d$ in Figure 2. The first term on the right side of Eq. (15) represents the (positive) distance from $\overrightarrow{\mathbf{r}}_{\mathbf{d}}$ to that same point. The required distance $g$ is the difference of these terms, so the minus sign is chosen in evaluating Eq. (15).

We now specialize to the case when the point $\left(x_{d}, y_{d}\right.$, $z_{d}$ ) is actually the detector point. Let it lie on the $y$ axis "behind" the cylinder, so the point becomes $\left(0,-r_{d}, z_{d}\right)$ and any ray from there to the cylinder points in the positive $y$ direction, so $v$ is always positive. Let the cylinder radius $R$ be $r_{c}$, the radius of the sphere that will be transformed to the cylinder of the same radius (akin to the $r_{p}$ of Sec. 2). Equation (15) becomes

$$
g=\frac{\left(v r_{d}\right)-\sqrt{\left(v r_{d}\right)^{2}-\left(r_{d}^{2}-r_{c}^{2}\right)\left(1-w^{2}\right)}}{\left(1-w^{2}\right)}
$$

Now for a spherical coordinate system centered at $(0$, $-r_{d}, z_{d}$ ), with the polar angle $\theta$ measured from the vector from there parallel to the $y$ axis (see Figure 2) and the azimuthal angle $\varphi$ measured from a vector parallel to the

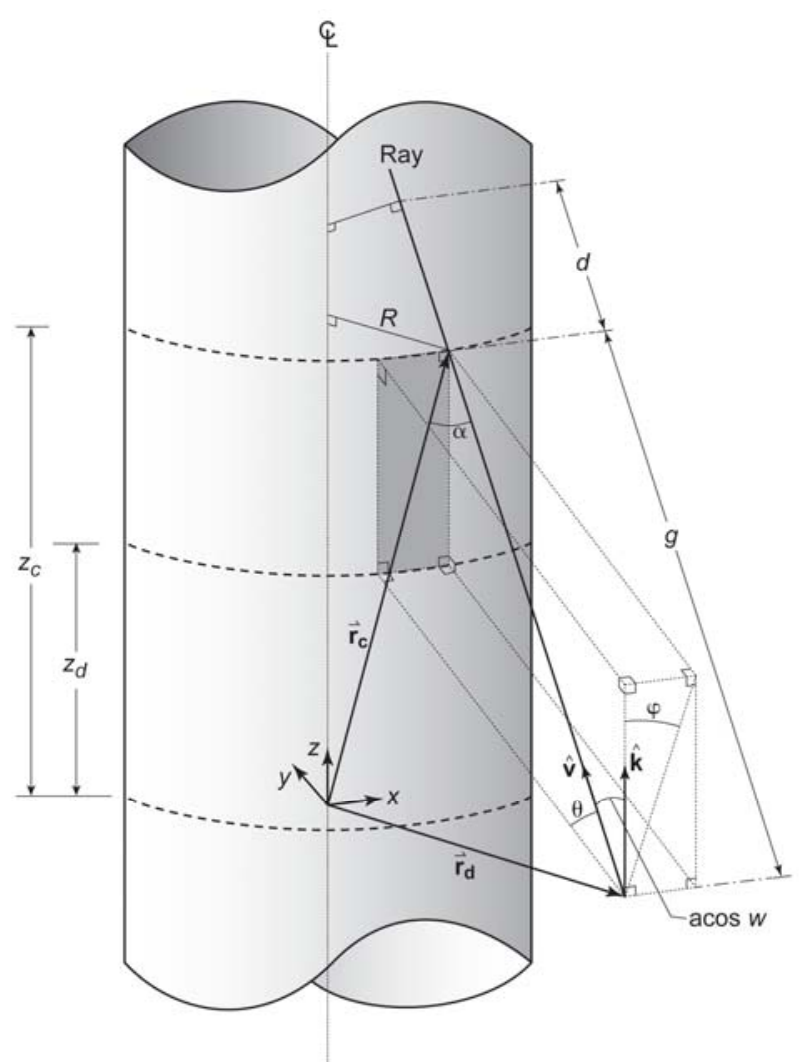

Figure 2. Geometry for the sphere-to-cylinder correction. 
axis of the cylinder (the $z$ axis), we have

$$
\left\{\begin{array}{l}
u=\sin \theta \sin \varphi \\
v=\cos \theta \\
w=\sin \theta \cos \varphi
\end{array}\right.
$$

The cylinder is infinite in axial extent. Substituting for $v$ and $w$ in Eq. (16) yields

$$
\begin{gathered}
g=\frac{\left(r_{d} \cos \theta\right)}{\left(1-\sin ^{2} \theta \cos ^{2} \varphi\right)} \\
-\frac{\sqrt{\left(r_{d} \cos \theta\right)^{2}-\left(r_{d}^{2}-r_{c}^{2}\right)\left(1-\sin ^{2} \theta \cos ^{2} \varphi\right)}}{\left(1-\sin ^{2} \theta \cos ^{2} \varphi\right)}
\end{gathered}
$$

The distance $h$ from the detector point to the sphere of radius $r_{c}$ centered within the cylinder is $c+b$ from Sec. 2 (Figure 1), which, by rearranging Eqs. (1) and (4) (and using $r_{c}$ instead of $r_{p}$ ), is

$$
h=r_{d} \cos \theta-\sqrt{r_{c}^{2}-r_{d}^{2} \sin ^{2} \theta}
$$

The quantity $h-g$ is the (unsigned) length along the ray from the outside of the sphere to the inside of the cylinder, analogous to the $b$ of Sec. 2. The quantity analogous to $b+a$ of Sec. 2 is $d=(g+d)-g$ or, recognizing that $g+d$ here is the same as $a+b+c=r_{d} \cos \theta$ of Sec. 2 [Eq. (1)], and using Eq.

(18) for $g$,

$$
\begin{gathered}
d=r_{d} \cos \theta-\frac{\left(r_{d} \cos \theta\right)}{\left(1-\sin ^{2} \theta \cos ^{2} \varphi\right)} \\
+\frac{\sqrt{\left(r_{d} \cos \theta\right)^{2}-\left(r_{d}^{2}-r_{c}^{2}\right)\left(1-\sin ^{2} \theta \cos ^{2} \varphi\right)}}{\left(1-\sin ^{2} \theta \cos ^{2} \varphi\right)}
\end{gathered}
$$

Unfortunately, the length $d$ here depends on the azimuthal angle $\varphi$, which normally does not exist in spherical codes. One approach to approximating this length is to take the average value of $d$ over $\varphi$ for each $\theta$. This involves taking the average value of $g$ for each $\theta$, $\bar{g}(\theta)$, over the range of $\varphi, 0$ to $2 \pi$, and using an average value for $d, \bar{d}(\theta)$, defined by

$$
\bar{d}(\theta) \equiv r_{d} \cos \theta-\bar{g}(\theta)
$$

The symmetry of the problem allows the average of $g$ to be taken over one-quarter of the range:

$$
\begin{gathered}
\bar{g}(\theta)=\frac{1}{\pi / 2} \int_{0}^{\pi / 2} d \varphi\left[\frac{\left(r_{d} \cos \theta\right)}{\left(1-\sin ^{2} \theta \cos ^{2} \varphi\right)}\right. \\
\left.-\frac{\sqrt{\left(r_{d} \cos \theta\right)^{2}-\left(r_{d}^{2}-r_{c}^{2}\right)\left(1-\sin ^{2} \theta \cos ^{2} \varphi\right)}}{\left(1-\sin ^{2} \theta \cos ^{2} \varphi\right)}\right]
\end{gathered}
$$

We rewrite Eq. (22) as

$$
\bar{g}(\theta)=\bar{g}_{1}(\theta)-\bar{g}_{2}(\theta)
$$

with

$$
\bar{g}_{1}(\theta) \equiv \frac{1}{\pi / 2} \int_{0}^{\pi / 2} d \varphi \frac{\left(r_{d} \cos \theta\right)}{\left(1-\sin ^{2} \theta \cos ^{2} \varphi\right)}
$$

and

$$
\begin{gathered}
\bar{g}_{2}(\theta) \equiv \frac{1}{\pi / 2} \times \\
\int_{0}^{\pi / 2} d \varphi \frac{\sqrt{\left(r_{d} \cos \theta\right)^{2}-\left(r_{d}^{2}-r_{c}^{2}\right)\left(1-\sin ^{2} \theta \cos ^{2} \varphi\right)}}{\left(1-\sin ^{2} \theta \cos ^{2} \varphi\right)}
\end{gathered}
$$

We now evaluate $\bar{g}_{1}(\theta)$, first by rewriting it as

$$
\bar{g}_{1}(\theta) \equiv \frac{r_{d}}{\pi / 2} \int_{0}^{\pi / 2} d \varphi \frac{\sqrt{1-\sin ^{2} \theta}}{\left(1-\sin ^{2} \theta \cos ^{2} \varphi\right)}
$$

The integral has a closed-form solution [3]:

$$
\begin{aligned}
\bar{g}_{1}(\theta) & =-\frac{r_{d}}{\pi / 2} \frac{\sqrt{1-\sin ^{2} \theta}}{\sqrt{\sin ^{2} \theta-1}}\left[\tanh ^{-1}\left(\frac{\tan \varphi}{\sqrt{\sin ^{2} \theta-1}}\right)\right]_{0}^{\pi / 2} \\
& =\frac{r_{d}}{\pi / 2} i\left[\tanh ^{-1}\left(-i \frac{\tan \varphi}{\cos \theta}\right)\right]_{0}^{\pi / 2}
\end{aligned}
$$

where we recognize that $\sqrt{\sin ^{2} \theta-1}=i \cos \theta$. Using the definition of the inverse hyperbolic tangent [4],

$$
\tanh ^{-1}(z)=\frac{1}{2}[\ln (1+z)-\ln (1-z)]
$$

where $z$ is a complex number, Eq. (27) becomes

$$
\bar{g}_{1}(\theta)=\frac{r_{d}}{\pi / 2} \frac{i}{2}\left[\ln \left(1-i \frac{\tan \varphi}{\cos \theta}\right)-\ln \left(1+i \frac{\tan \varphi}{\cos \theta}\right)\right]_{0}^{\pi / 2}
$$

Using the properties of natural logarithms of imaginary numbers, Eq. (29) can be written

$$
\begin{gathered}
\bar{g}_{1}(\theta)=\frac{r_{d}}{\pi / 2} \frac{i}{2}\left[\ln \left(\sqrt{1+\frac{\tan ^{2} \varphi}{\cos ^{2} \theta}}\right)\right. \\
-i \operatorname{acos}\left(\sqrt{1+\frac{\tan ^{2} \varphi}{\cos ^{2} \theta}}\right)-\ln \left(\sqrt{1+\frac{\tan ^{2} \varphi}{\cos ^{2} \theta}}\right) \\
-i \operatorname{acos}\left(1 / \sqrt{\left.\left.1+\frac{\tan ^{2} \varphi}{\cos ^{2} \theta}\right)\right]_{0}^{\pi / 2}}\right. \\
=\frac{r_{d}}{\pi / 2}\left[\operatorname{acos}\left(1 / \sqrt{1+\frac{\tan ^{2} \varphi}{\cos ^{2} \theta}}\right)\right]_{0}^{\pi / 2} \\
=\frac{r_{d}}{\pi / 2}\left[\operatorname{acos}\left(\frac{\cos \theta \cos ^{2}}{\sqrt{\cos ^{2} \theta \cos ^{2} \varphi+\sin ^{2} \varphi}}\right)\right]_{0}^{\pi / 2}
\end{gathered}
$$

Now using the integral limits yields the simple result

$$
\bar{g}_{1}(\theta)=\frac{r_{d}}{\pi / 2}[\operatorname{acos}(0)-\operatorname{acos}(1)]=r_{d}
$$

Using Eq. (23) in Eq. (21) and using Eqs. (31) and (25) for $\bar{g}_{1}(\theta)$ and $\bar{g}_{2}(\theta)$ (and factoring out an $r_{d}$ ) yields

$$
\begin{gathered}
\bar{d}(\theta)=r_{d}(\cos \theta-1)+ \\
\frac{r_{d}}{\pi / 2} \int_{0}^{\pi / 2} d \varphi \frac{\sqrt{\cos ^{2} \theta-\left(1-r_{c}^{2} / r_{d}^{2}\right)\left(1-\sin ^{2} \theta \cos ^{2} \varphi\right)}}{\left(1-\sin ^{2} \theta \cos ^{2} \varphi\right)}
\end{gathered}
$$

In our implementation, the integral in Eq. (32) is evaluated with Simpson's rule with interval refinement [5]. 


\section{Results}

The conversion of a spherical shield surface to a flat shield surface or a curved cylindrical surface within a one-dimensional spherical ray-tracing code [2] was tested numerically using a 10-kg highly enriched uranium sphere $\left(94.73 \%{ }^{235} \mathrm{U}, 5.27 \%{ }^{238} \mathrm{U}\right.$ by weight; density $18.74 \mathrm{~g} / \mathrm{cm}^{3}$; radius $5.03169 \mathrm{~cm}$ ) centered within a stainless steel (density $7.86 \mathrm{~g} / \mathrm{cm}^{3}$ ) box (flat walls) or can (cylindrical walls) with walls $1 \mathrm{~cm}$ thick. Reference results were computed using MCNP [6] with the multidimensional geometries and a point detector scoring uncollided photons only. The detector is $100 \mathrm{~cm}$ from the center of the source. The errors in the corrected spherical ray-tracing, relative to the reference results, for a low-energy gamma-ray line from uranium are shown in Figure 3. These are the diamonds and squares with values close to 0 . The effect of using an average value of the azimuthal angle is seen when the cylindrical shield is close to the source. These errors are compared with the errors made when the box and cylinder shields are assumed to be spherical for purposes of spherical ray-tracing, which are $27 \%$ and $16 \%$, respectively, when the shield is close to the source. Correcting for nonspherical shields is less important when the shield is far from the source.

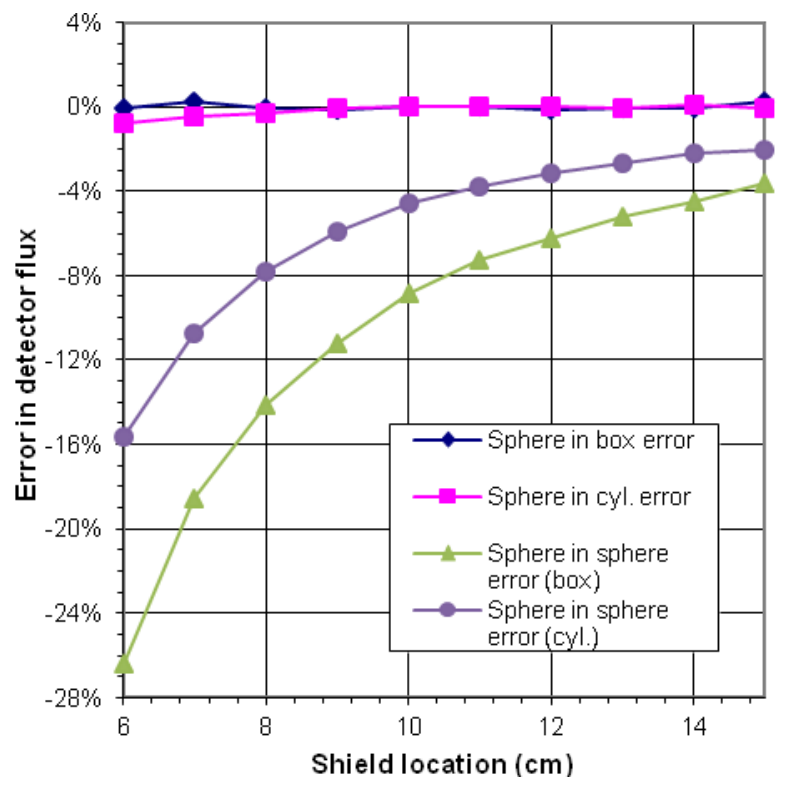

Figure 3. Error in the $144-\mathrm{keV}$ line flux for the spherical source in a box and a cylindrical can with wall thickness $1 \mathrm{~cm}$.

\section{Conclusion}

Spherical ray-tracing for uncollided photon fluxes is very fast, but it is often not appropriate to be used when shielding is flat or cylindrical, even when the source is spherical. In this paper, corrections are derived for spherical ray-tracing codes to account for flat or cylindrical shielding. The corrections are made only to the shielding, not the source; only for the outermost layers of shielding; and only for the case when a line connecting the detector with the center of the source is orthogonal to the shielding. The equations could be corrected to account for non-orthogonal cases, but the sphere-to-flat correction would then be approximate, not exact, if the average-azimuthal-angle method were used.

Obviously, spherical ray-tracing cannot accommodate every situation the analyst faces. As the problem gets more complex, eventually a more exact geometric treatment will be necessary. However, if the source is spherical and it is important to account for flat or cylindrical shielding, then the methods of this paper can be implemented.

\section{Acknowledgements}

The author is grateful to Ms. P. Paine, Los Alamos National Laboratory, for drawing Figures 1 and 2.

\section{References}

[1] A.B. Chilton, J.K. Shultis and R.E. Faw, Principles of Radiation Shielding, Prentice Hall, Inc., Edgewood Cliffs, New Jersey (1984), pp. 155-175, ISBN 0-13-709907-X.

[2] J.A. Favorite, K.C. Bledsoe and D.I. Ketcheson, Surface and volume integrals of uncollided adjoint fluxes and forward-adjoint flux products, $\mathrm{Nucl}$. Sci. Eng. 163 (2009), pp. 73-84.

[3] Wolfram Mathematica on-line integrator; integrals.wolfram.com

[4] Staff of Research and Education Association, Handbook of Mathematical, Scientific, and Engineering Formulas, Tables, Functions, Graphs, Transforms, Research and Education Association, New York (revised 1986), p. 266, ISBN 0-87891-521-4.

[5] W.H. Press, S.A. Teukolsky, W.T. Vetterling and B.P. Flannery, Numerical Recipes in FORTRAN: The Art of Scientific Computing, $2^{\text {nd }}$ Ed. (reprinted with corrections), Cambridge University Press, (1994), pp. 130-133, ISBN 0-521-43064-X.

[6] X-5 Monte Carlo Team, MCNP - A General $N$-Particle Transport Code, Version 5 - Volume I: Overview and Theory, LA-UR-03-1987, Los Alamos National Laboratory, (2003). 